\title{
SURFACE PHOTOMETRY OF COMET P/ENCKE
}

\author{
S. DJORgovski AND HYRON SPINRAD ${ }^{\text {a) }}$ \\ Astronomy Department, University of California, Berkeley, California 94720 \\ Received 27 December 1984
}

\begin{abstract}
We have developed a scheme to clean cometary digital images from offending background-star trails, and applied this technique to a pair of deep Kitt Peak 4-m plates of comet P/Encke, taken in October 1980. Simultaneous and subsequent digital spectra have been obtained at Lick Observatory. The nonpolluted coma images show a strong asymmetric sunward-oriented fan/jet, and an extended and rounder (mostly gaseous) main coma, out to $\sim 10^{5} \mathrm{~km}$ radius. The stellar-trail point-spread function has a narrow width $(\sigma \sim 0.6$ arcsec), so that spatial resolution better than $\sim 300 \mathrm{~km}$ is achieved at the comet. The photometric gradient near the nucleus is very steep, strongly suggesting an icy-grain component which evaporates quickly (at radii $\leqslant 500 \mathrm{~km}$ ) in the sunlight. Further from the nucleus, the profile becomes shallower, bluer, and more gas dominated. The effect of solar radiation pressure on $\mathrm{C}_{2}, \mathrm{CN}$, and other molecules is probably responsible for the rounding of the outer, faint isophotes. The source of the molecules is likely to be larger than the nucleus itself, and a substantial fraction may originate in the jet. The technique described here may also be applicable in surface photometry of galaxies, in cases where the heavy image pollution by foreground stars is present.
\end{abstract}

\section{INTRODUCTION}

In spite of the increasing scientific interest in comets, and the upcoming spacecraft missions to them, very little quantitative information is available about the distribution of light and color in these objects. Much progress has been made recently in spectroscopy (see, e.g., Newburn and Spinrad 1984, 1985). However, further advances in the interpretation of the spectroscopic data and modeling of comae require better knowledge of cometary optical morphology, and more quantitative surface photometry (cf. Jewitt et al. 1982).

The surface photometry of comets (and especially their faint, extended comae) is extremely difficult because of the presence of numerous bright background stars trailing across the image as the comet moves. This type of background can not be subtracted easily in any statistical sense. We have developed an automatic digital technique which enables us to effectively locate all the stellar trials down to the noise level in any panoramic detector data (in this case, digitized photographic plates). After this "cleaning", we can use the nonpolluted data to do the surface photometry of cometary envelopes down to very faint levels. In fact, our photometry is limited by the plate calibration uncertainties and the large-scale plate sensitivity and fog variations approximately on the level of $2 \%-3 \%$ of the sky, or roughly $\sim 24 \mathrm{mag} / \operatorname{arcsec}^{2}$.

\section{THE DATA ON COMET P/ENCKE}

One IIIa-J/GG-385 plate (baked $2 \mathrm{hr}$ in the forming gas), taken at 1980 October 10 (mid-exposure time: $11^{\mathrm{h}} 44^{\mathrm{m}} \mathrm{UT}$ ), and one baked IIIa-F/GG-495 plate taken at 1980 October $9\left(11^{\mathrm{h}} 43^{\mathrm{m}} \mathrm{UT}\right), 58$ days before the comet's perihelion passage, were obtained at the prime focus of the Kitt Peak 4-m telescope. The exposure times were 30 and $20 \mathrm{~min}$, respectively. The seeing on these plates was very good; we have determined an approximate point-spread function (PSF) perpendicular to the star trails. The $F$ plate has a PSF

\footnotetext{
a) Visiting Astronomer, Kitt Peak National Observatory, National Optical Astronomy Observatories, Operated by the AURA, Inc., under contract with the NSF.
}

HWHM of $0.6 \pm 0.1 \mathrm{arcsec}$, and the $J$ plate has a PSF HWHM of $0.8 \pm 0.1$ arcsec. The comet was then at $1.24 \mathrm{AU}$ from the Sun, and $0.47 \mathrm{AU}$ from the Earth, so that 1 arcsec on the sky is $341 \mathrm{~km}$ (in projection) at the comet.

The plates were digitized on the Berkeley PDS microdensitometer with $20 \times 20-\mu$ square pixels, and calibrated by the standard procedures. The $J$ plate was digitized in a $2048^{2}$ raster, and the $F$ plate in a $1024^{2}$ raster. After the star removal as described here, digital photometry was performed in concentric annular apertures for the four quadrants, as shown in Fig. 1.

Digital spectra of the nucleus and coma of $P$ /Encke were obtained simultaneously and again a month later with the Lick ITS spectrograph. Shown here in Fig. 2 is a composite spectrum of the nuclear region, demonstrating its strong emission activity. Note that the Swan carbon bands dominate well over the solar continuum in the $J$ (blue/green) band; thus the very blue color of the coma. In the $F$ (red) band, weaker $\mathrm{C}_{2}$ and weak [ $\mathrm{O}_{\mathrm{I}}$ ] lines are seen, but at least near the nucleus, the continuum should dominate the red light.

\section{THE STAR REMOVAL PROCEDURE}

The data scans contain numerous stellar trails, in the direction of the comet's apparent motion to the $\mathrm{NE}$ (which is along the main diagonal of our data frames). It was necessary to find all those pixels which are suspected of belonging to a star trail, and ignore them while doing photometry. The procedure which we have adopted to accomplish this goal may be of some interest to other observers, and we will describe it in some detail.

First, a smooth, star-free background was obtained through a combination of median filtering and smoothing, and substracted from the data. (Notice that nonparametric filtering is not flux-preserving, so that this background can not be used for photometry by itself.) The remaining frame contains the star trails and the cometary nucleus. Those features were then enhanced with a directional gradient technique, in the direction perpendicular to the trails. This is accomplished by convolution with a kernel of the form: 


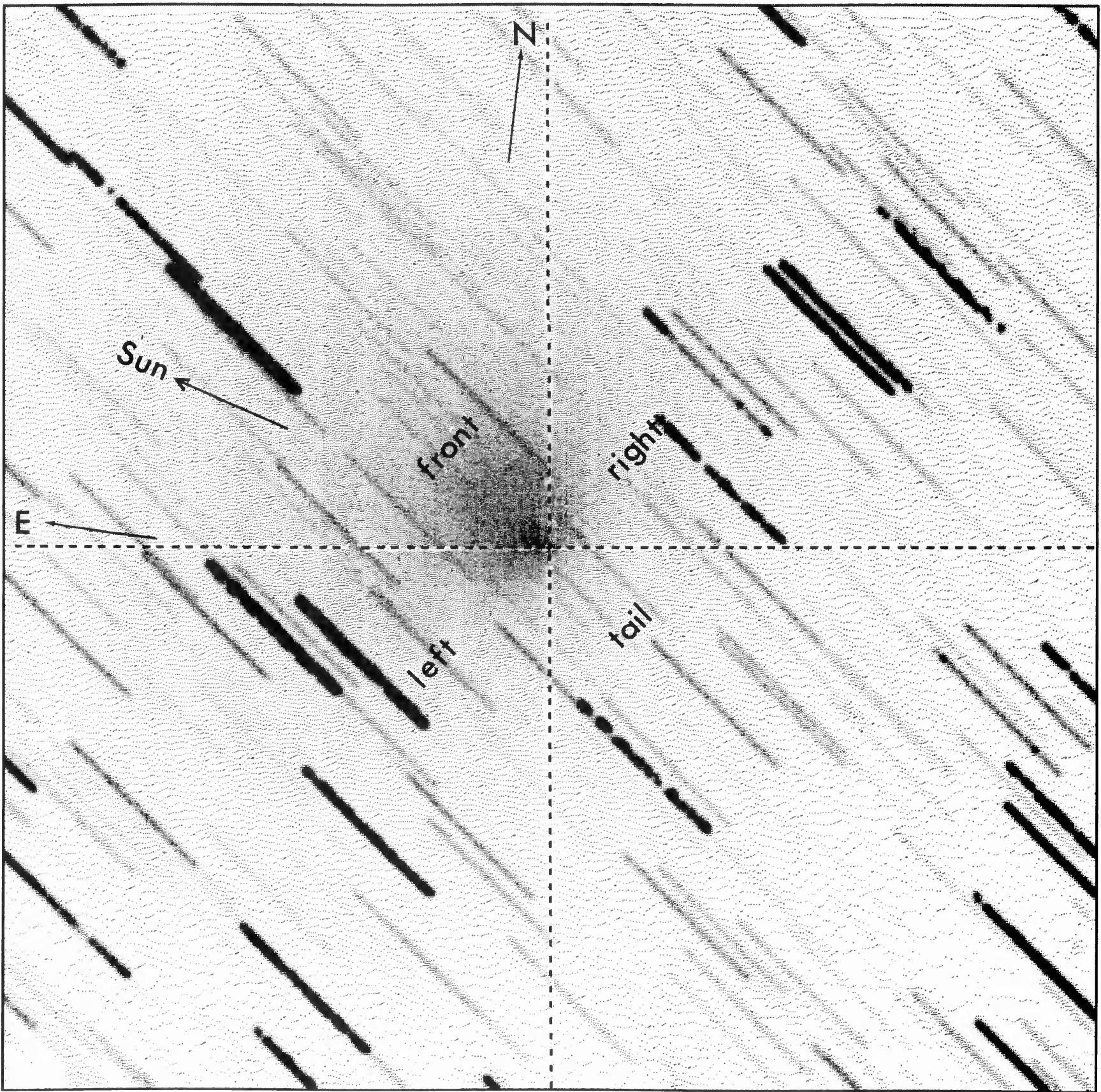

FIG. 1. Linear intensity image of $\mathrm{P} /$ Encke, from the $J$ plate. The field is $\sim 12.7 \mathrm{arcmin}^{2}$. The orientation and quadrants notation are indicated. The comet's apparent motion was from SW to NE.

$$
k=\begin{array}{rrrrr}
0 & 0 & 0 & 0 & 0 \\
0 & 0 & 1 & 1 & 1 \\
0 & -1 & 0 & 1 & 1 \\
0 & -1 & -1 & 0 & 1 \\
0 & -1 & -1 & -1 & 0
\end{array}
$$

which is equivalent to presmoothing, diagonal offset, and self-subtraction. The absolute value of the resulting image was then taken. This technique stongly enhances all linear features in the direction of the main diagonal, but it leaves a trench along the middle of each star trail, since the derivatives are zero at the peaks. This is amended by making a linear combination of this frame and the background-subtracted frame, with additional smoothing. A directional gradient across the star trails is preferred to the two-dimensional (or Laplacian) gradient, since the latter one would differentiate over some unnecessary noise. The resulting frame is then truncated at some safe limit above the noise, and remapped so that the pixels above the threshold (which, presumably, belong to the stars) have a flag value (1, say), and the remaining ones are set to zero. The final touch is to remove "by hand" the cometary nucleus from this "mask" frame, since it will also get enhanced.

The flagged pixels are simply ignored while doing photometry. We preferred to do it in a conservative way, and 


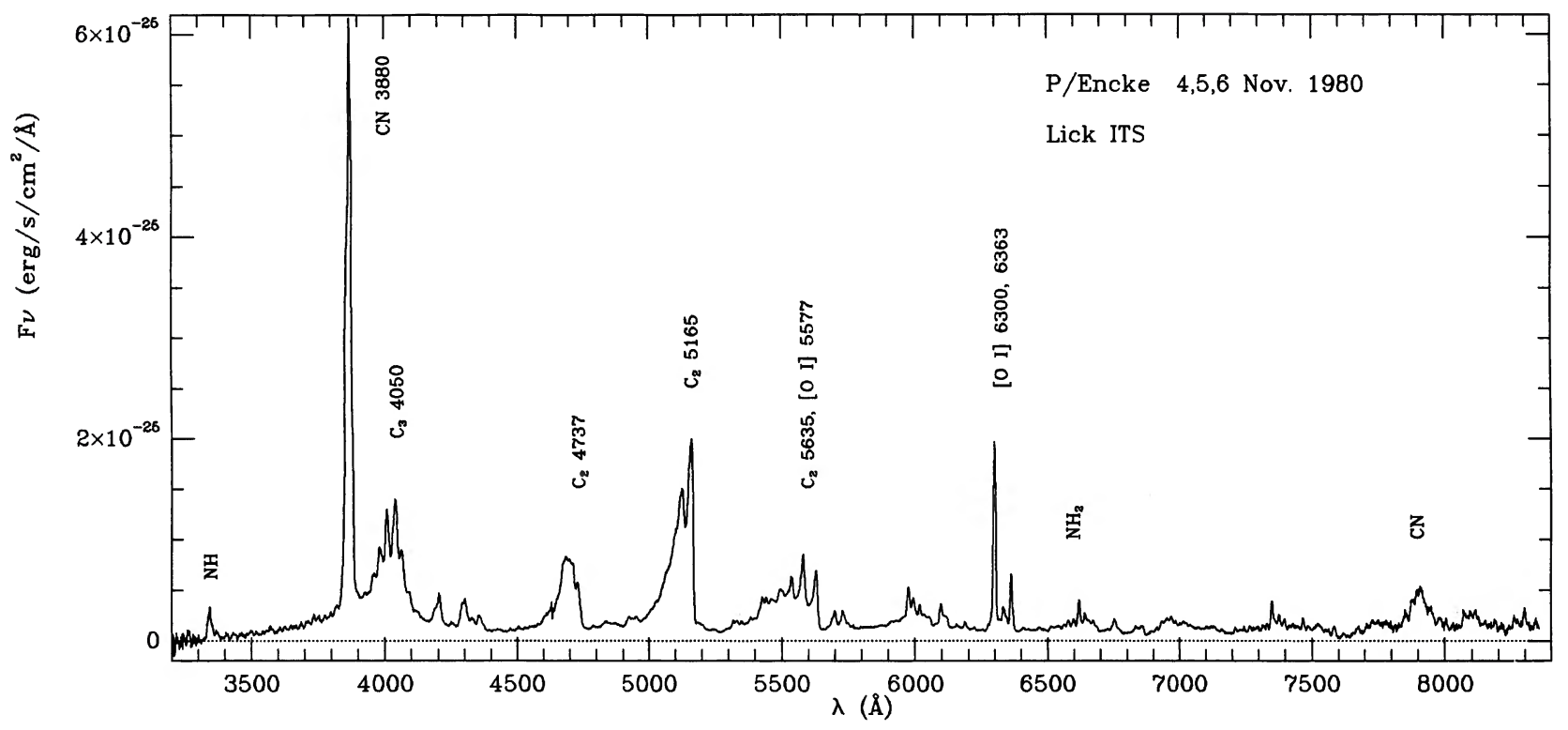

FIG. 2. Spectrum of the near-nucleus coma of P/Encke, obtained at Lick in 1980 November, about 20 days after the photometric plates were taken. The blue ( $J$ plate) continuum is dominated by the molecular emission bands.

ended up flagging about $25 \%$ of the data; the scan is so large that this does not impoverish the photometry. One caveat applies: this procedure will miss the faintest stars, which are at the plate noise level or fainter, but this turns out not to be a problem.

We can now interpolate over the flagged regions, in order to produce a star-free comet picture, and investigate the shape of the cometary coma at larger scales. This was done by least-squares fitting to the "good" points which surround flagged pixels. The interpolation was done first in rows and columns separately, and the two resulting pictures were coadded. The least-squares interpolation can be unstable if there are few constraining points. This is the cause of any residual streakiness, or a slight scratchy pattern in the final picture. The pictures of the comet before and after star removal, and their difference, are shown in Fig. 3. Notice the decreasing asymmetry of the coma far from the nuclear condensation of the comet.

\section{THE PHOTOMETRIC PROCEDURE}

The calibration spots on each plate were scanned several times before and after the comet scans, and averaged. This multiple spot scanning compensates for the slight gain drifts in the PDS amplifier, which occur on the time scales of a couple of minutes, and thus may systematically bias the individual spot scans; such drifts average out in much larger and longer comet scans. The $\log (I)$ values were obtained by interpolating data published by Schweizer, Gonzales, and Saa (1980) for the $\lambda_{\text {eff }}=4880 \AA$ (IIIaJ + GG-385) and $\lambda_{\text {eff }}$ $=6000 \AA$ (IIIaF + GG-495). The HD curve was obtained by fitting semi-interactively to the formula

$$
\log (I)_{i}=\frac{P^{n}\left(D_{i}\right)}{\left(D_{i}-D_{\text {toe }}\right) \cdot\left(D_{i}-D_{\text {sat }}\right)},
$$

where $(D)_{i}$ and $\log (I)_{i}$ are the mean density and the $\log$ in-
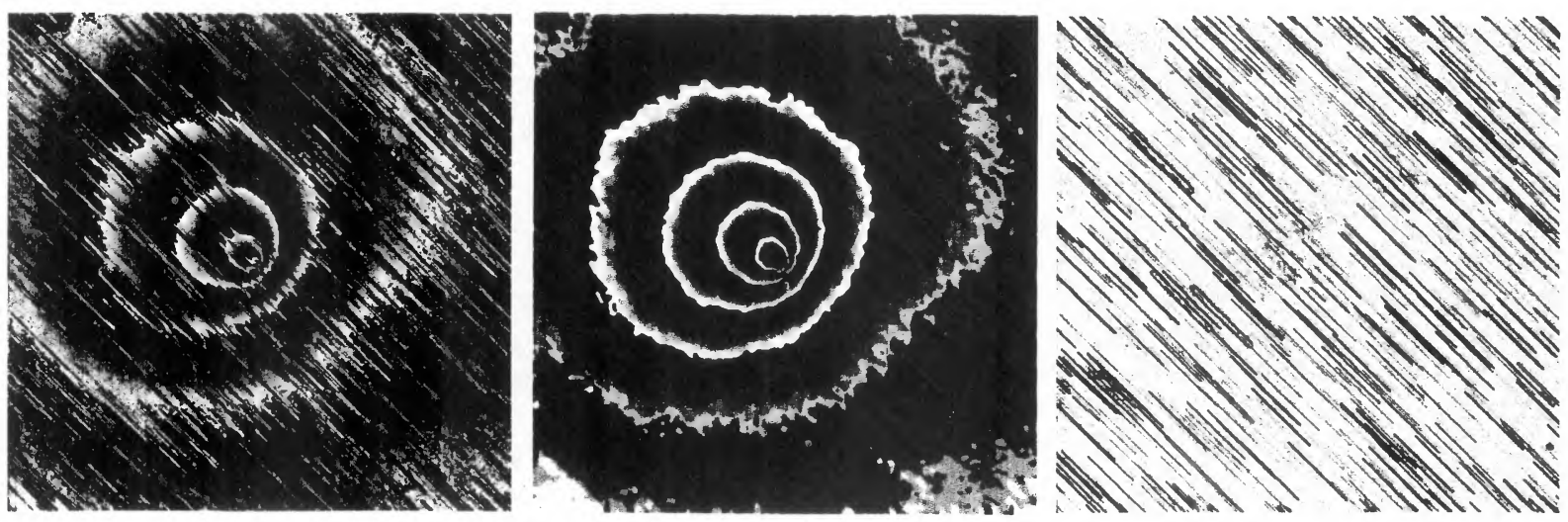

FIG. 3. (Left) the $J$ image of P/Encke, including the background stellar trails, in logarithmic representation, with one gray-scale cycle per magnitude interval. (Center) the same image, after the star removal and interpolation, as described in the text; note the "bump" in the outer isophotes. (Right) the difference between the raw and cleaned images, showing the background stellar trails. The size and orientation are as in Fig. 1. 
tensity for the $i$ th spot, $D_{\text {toe }}$ and $D_{\text {sat }}$ asymptotic densities in the HD curve, and $P^{n}\left(D_{i}\right)$ an $n$ th-order polynomial of $D_{i}(n$ is typically 5 or 7 ). This procedure is very user-friendly and reliable, and produces calibration errors (in intensity) of the order of $1 \%-2 \%$ in the toe, and less than $0.1 \%$ in the linear part of the HD curve.

We have divided each of the comet scans into four quadrants, "front", "tail", "left", and "right" (as schematically shown in Fig. 1), each having the central cometary nucleus pixel in its appropriate corner. The photometry was done in a series of concentric annular apertures centered on the nucleus, and spaced logarithmically. The scale differences between the $J$ and the $F$ plates $(18.59$ and $18.74 \mathrm{arcsec} / \mathrm{mm}$, respectively) were compensated so that the annuli had different radii in the pixels, but the same radii in arcseconds. For a given annulus, we compute the effective radius as an areaweighted mean:

$$
r_{i}=\frac{2 \cdot\left(a_{i+1}^{3}-a_{i}^{3}\right)}{3 \cdot\left(a_{i+1}^{2}-a_{i}^{2}\right)},
$$

where $r_{i}$ is the effective radius for the $i$ th annulus, and $a_{i+1}$ and $a_{i}$ its limiting radii.

The effective surface brightness in each annulus was computed in two different ways. In the first method, we converted density for each nonflagged pixel into intensity, added them up, and computed the mean. In the second method, we accumulated the density histograms of all nonflagged pixels for every annulus, and evaluated the median or the mode (the latter by fitting a parabola to the histogram peak). Those density values were then converted into the corresponding intensities. This method was designed to avoid the influence of unremoved stars, since they would contribute to the high tail of the histogram. We choose density, rather than intensity, histograms for two reasons: first, the intensity histograms are sparse, because of the nonlinearity of the density-to-intensity conversion; second, the radial-density change within an annulus is very nearly linear, whereas the intensity change is not, and that may bias the mode estimate. Unfortunately, this method does not work so well when the total number of pixels in an annulus is small. Where it does work, it agrees extremely well with the first, summation method, suggesting that the perturbations by unremoved faint stars are negligible. Thus, in order to maintain consistency throughout the profile, we use the summation photometry only, and keep the histogram photometry as an internal check. The general reliability of our photometric scheme was tested both on Monte Carlo realizations of artificial data, and PDS scans of stars for which photoelectric photometry was available.

The sky surface-brightness estimates were obtained from the density histograms of a number of star-free spots, as distant from the cometary nucleus as possible (far beyond the limit of our data scans). The sky spot scans were repeated in the same manner and with the same purpose as the calibration spot scans. Corresponding sky intensity values were then subtracted from the comet surface-brightness profiles. As a rough zero-point calibration, we can use the estimates for the Kitt Peak sky brightness, viz., $\mu_{J} \cong 22.5$ and $\mu_{F}$ $\cong 21.5 \mathrm{mag} / \operatorname{arcsec}^{2}$.

In order to obtain some quantitative information about the change of isophotal shapes, we also did the surface photometry with an iterative ellipse-fitting program, with the isophote centers allowed to vary. This was done with an algorithm similar to the one described by Kent (1983).
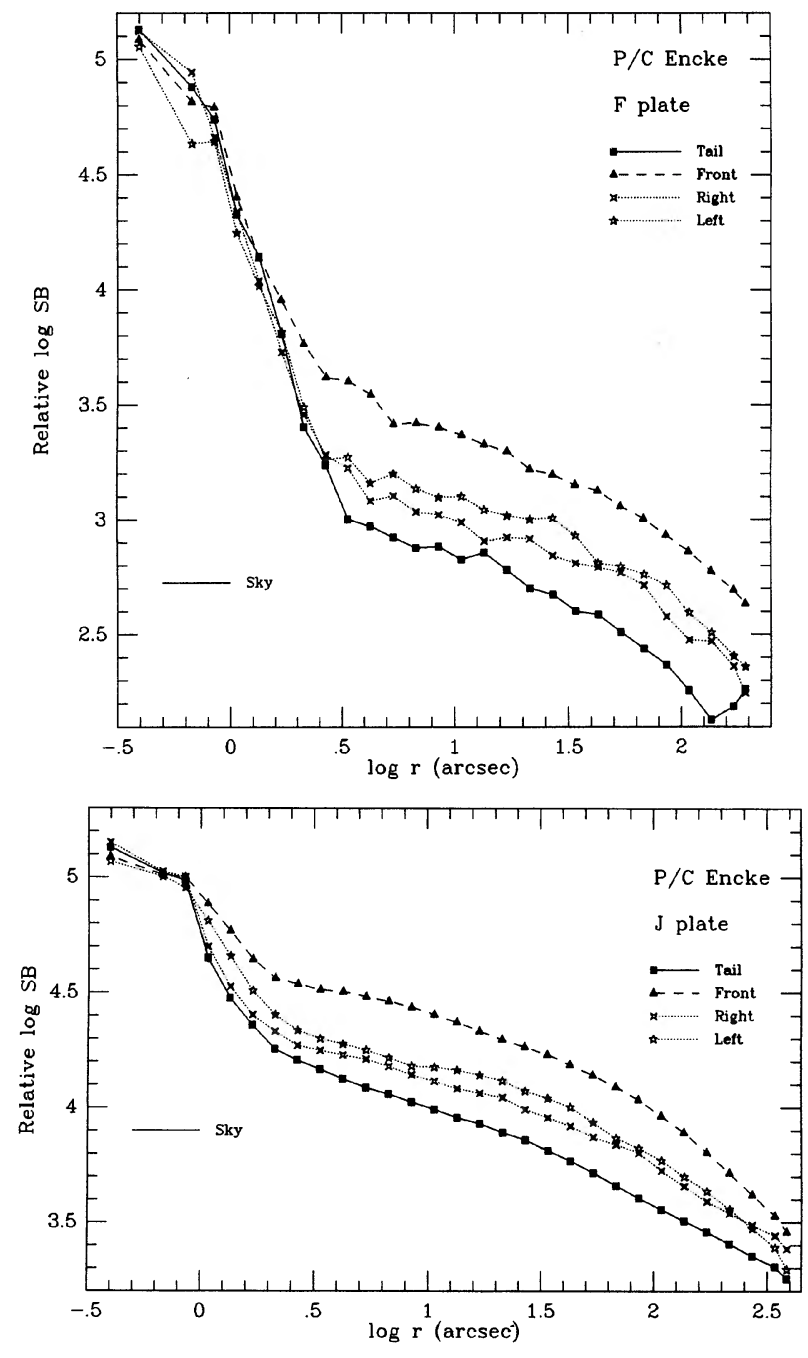

FIG. 4. Surface-brightness profiles in $\log -\log$ representation, for the $F$ plate (top), and the $J$ plate (bottom). The profiles for different quadrants are shown, as marked in Fig. 1.

\section{PHOTOMETRIC RESULTS AND DISCUSSION}

The surface brightness profiles in $J$ and $F$ are shown here in $\log -\log$ (Fig. 4) and log-linear (Fig. 5) plots. The $(J-F)$ color plot is shown in Fig. 6, and the ellipticity profiles in Fig. 7. The light-profile morphology can be described as follows:

-There is an unresolved "nucleus" (scale $\sim 1$ arcsec, or $300 \mathrm{~km}$ ). It is completely unresolved on the $F$ ( red) plate (seeing HWHM $\sim 0.6$ arcsec), but on the $J$ (green) plate (seeing HWHM $\sim 0.8$ arcsec), the power-law decline in surface brightness sets in after $\sim 1$ arcsec.

-Following the nucleus $(r \geqslant 3$ arcsec) is a shallow powerlaw decline of surface brightness, with the slope $\sim-0.5$, extending up to $\sim 20-30$ arcsec (or $7000-10000 \mathrm{~km}$ at the comet) (Fig. 4). This is unexpectedly shallow, since the evaporization models predict a slope of $\sim-1$ (in projection).

-Following that, the surface brightness declines exponentially, with azimuthally variable $e$-folding length, ranging from $\sim 100$ to $\sim 300$ arcsec (Fig. 5). This exponential decline may be understood as being due to the particles expanding 


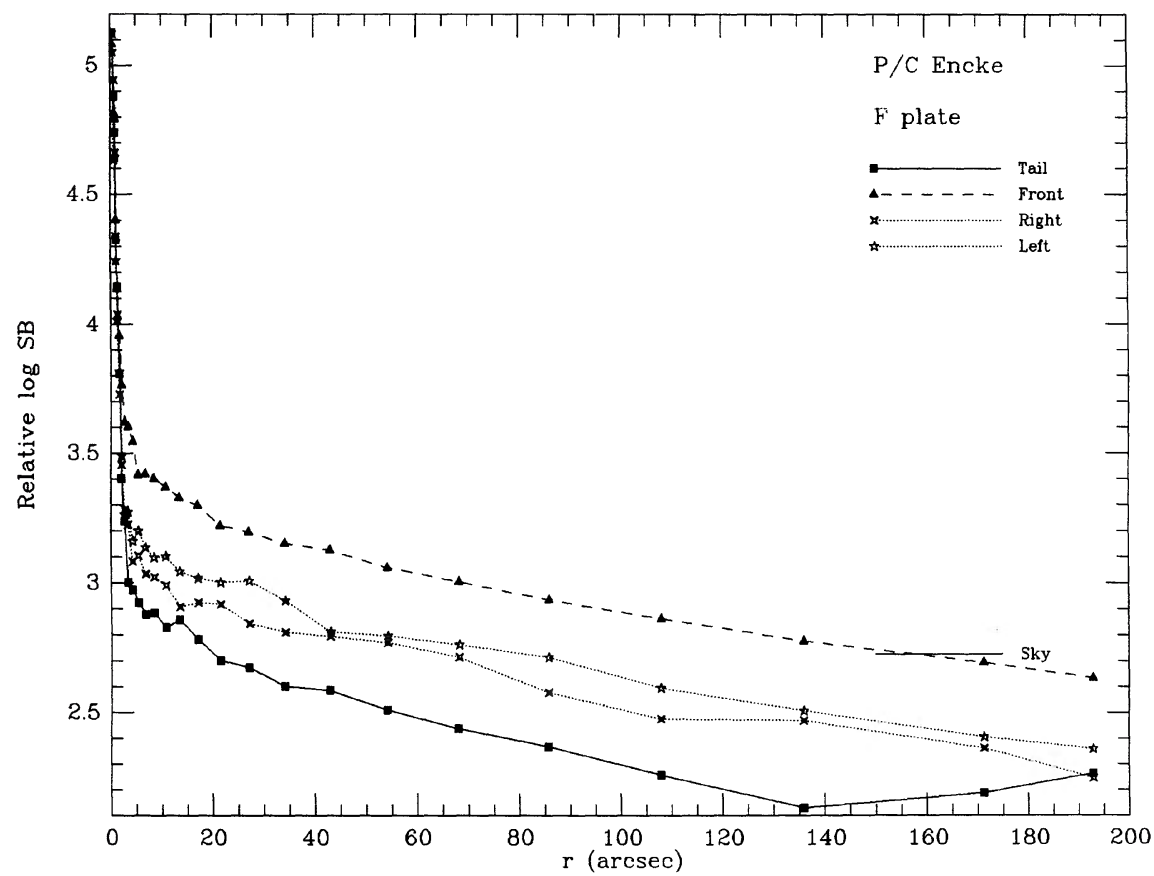

FIG. 5. Same as in Fig. 4, but in log-linear representation. The exponential surfacebrightness decline in the outer coma is apparent.

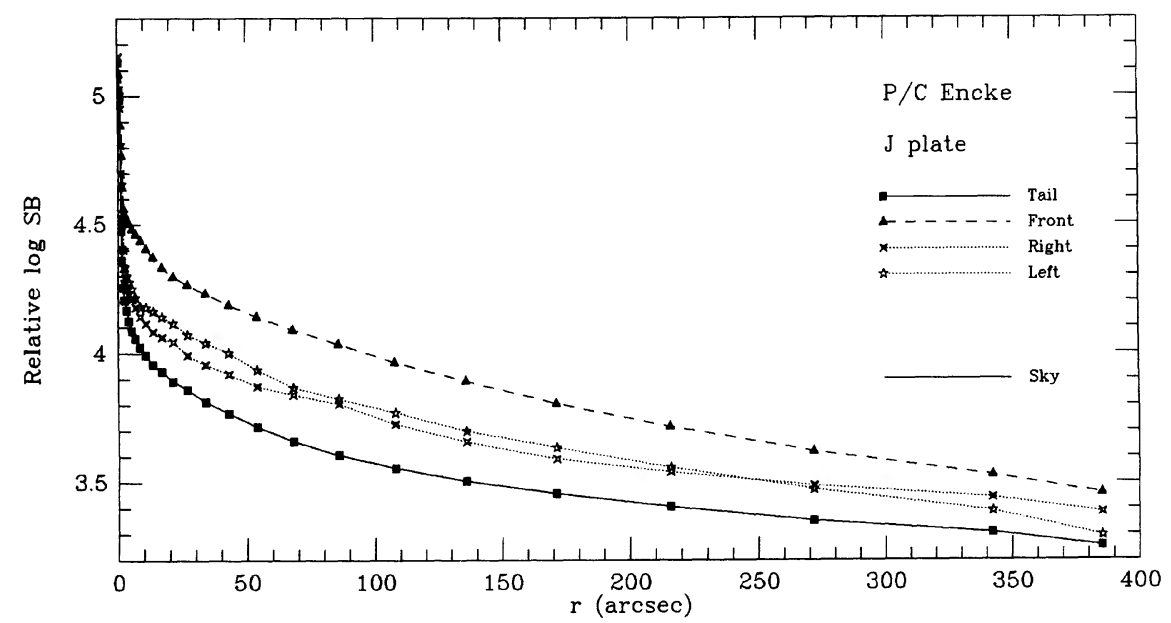

radially outward with a uniform (thermal?) velocity, and with the decay probability constant in time. The hydrodynamical (solar radiation pressure) effects are probably responsible for the azimuthal variation of the $e$-folding length.

The color profile (Fig. 6) shows a strong color gradient in the nucleus, partly due to the seeing differences, and partly due to the power-law envelope setting in. The outer coma is very blue, reflecting the prevalence of molecular carbon bands over the reflected solar continuum. The red nucleus is apparently dust dominated. Note also that the outer radial color gradients are very shallow, if present at all. There is a very notable azimuthal color variation in the sense of the front/fan/Sun side being the reddest (least $\mathrm{C}_{2}$ emission). $\mathrm{Re}$ call that the (blue) $J$ plate is dominated by the light from the molecular emission bands, whereas the (red) $F$ plate is mostly dominated by the reflected solar continuum, especially at the small radial distances from the nucleus.

P/Encke has long been known to display a broad, fan- shaped coma, emanating from the nucleus in the general direction of the Sun. Presumably, the fan detected on our photographs represents an anisotropic release of dust and gas from the solar-illuminated hemisphere, or a "hotspot" source within it; according to Sekanina (1979), this is the Northern hemisphere at preperihelion times. Whipple and Sekanina (1979) discuss the likelihood that Encke's Southern hemisphere is now largely devoid of volatile material, since it consistently sees the Sun just past the perihelion $(q=0.34$ $\mathrm{AU})$. We can use our photometry to explore the outgassing from the sunlit hemisphere of the comet, at the epoch of our plates ( 58 days before the perihelion, in its 1980 apparition). It may be that we are here dealing with yet another manifestation of an astrophysical jet, and on the smallest scale so far.

In Fig. 1 , the $J$ plate of $P$ /Encke, the solar position angle (P.A.) is $73^{\circ}$; the P.A. of the fan is $\sim 50^{\circ}$. Thus, the projected lag angle is about $23^{\circ}$. This is similar to the fan P.A. in the P/ Encke 1947 apparition (Sekanina 1979). 


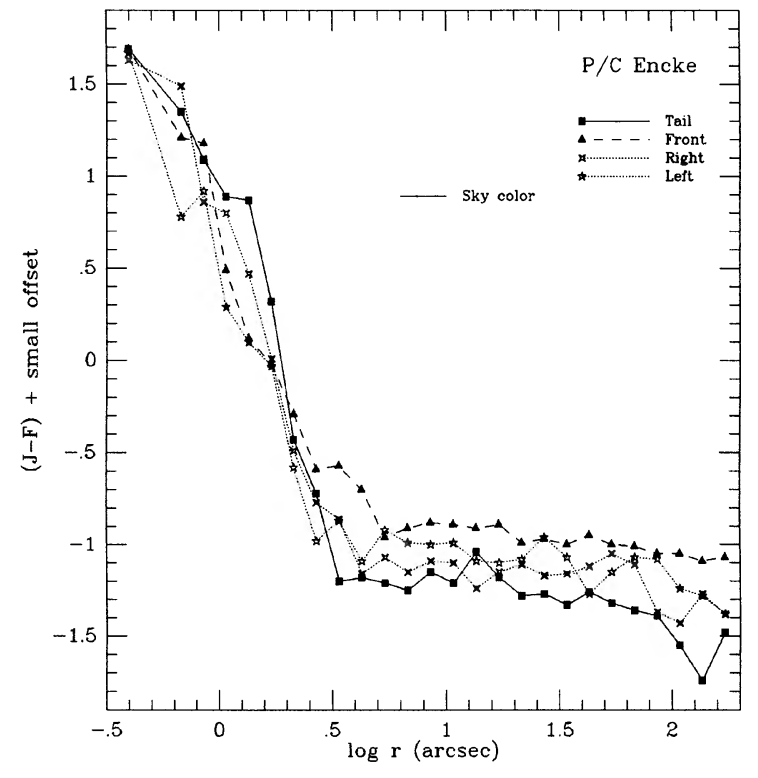

FIG. 6. The color profiles for the four quadrants, normalized with the sky color. Note the azimuthal color gradient in the coma: blue color implies strong gaseous emission.

Our discussion now centers on the comet's radial profile in the fan direction (the "front" quadrant). The most striking feature of the radial light distribution on the $F$ plate is the very rapid falloff within the innermost 2 arcsec. Beyond that radius, the distribution flattens markedly. The shallow surface-brightness decline on both plates at radii $\geqslant 10$ arcsec is presumably due to the creation of daughter radicals $\left(\mathrm{C}_{2}, \mathrm{CN}\right)$ in the expanding coma, and their exponential decay by photodissociation.

Our main point is that the $F$ plate surface-brightness decline in the jet has a power-law slope at the nucleus which is far steeper than anticipated for refractory grains, with a possible contribution from $\mathrm{C}_{2}$ and $\mathrm{NH}_{2}$ emission. Both of these would normally yield an $r^{-1}$ radial light profile, although only in a narrow zone (cf. Delsemme and Miller 1971; and Jewitt et al. 1982). From $\sim 1$ to $\sim 2$ arcsec in the jet direc-

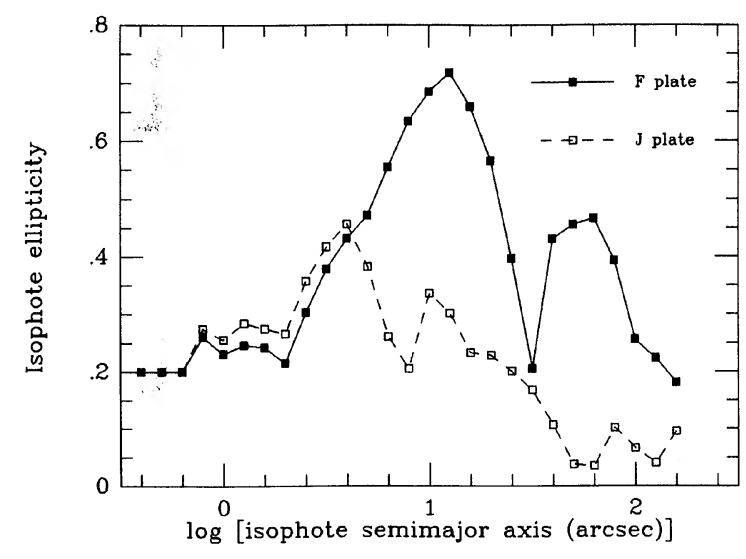

FIG. 7. Ellipticity profiles for the isophotes in the two plates. The maximum corresponds to the jet/fan feature, which stands out more on the $F$ plate. The isophote centers are allowed to vary. tion, the observed slope is at least -2 , and this limit value is determined largely by the seeing. This steep decline in the surface brightness is rather similar to that observed by Jewitt et al. (1982) in the inner 1 arcsec of their comet P/StephanOterma image. The icy-grain hypothesis works moderately well for P/Encke; this is especially attractive because Newburn and Spinrad (1985) have found from the "dust"-to-gas mass ratios derived from nuclear spectroscopy that $P$ /Encke had an anomalous decrease in its grain "mass loading" as the comet approached the Sun in its 1980 apparition. They note that for comet $\mathbf{P} /$ Tuttle, the mass load is roughly constant with heliocentric distance, the ratio being $\sim 0.08$ throughout, whereas for the $\mathrm{P} /$ Encke this ratio decreased by a factor of 16 (starting at the value 0.08 ) as the comet moved from heliocentric distance of 1.9 to $0.78 \mathrm{AU}$. Such a decrease is consistent with strong evaporation of icy grains in the innermost $1000 \mathrm{~km}$ (which corresponds to a typical spectroscopic observing diaphragm at the distance to the comet).

Beyond radii of several arcsec, the isophotes become increasingly rounder (Fig. 7). This must be governed by the two effects, the asymmetric sunward evaporation, and the solar radiation pressure. The latter will operate, although inefficiently, upon the few refractory grains, residual icy grains, and the daughter radicals $\mathrm{C}_{2}$ and $\mathrm{CN}$, which dominate the light away from the nucleus, especially in the $J$ band (some, but not all, $\mathrm{CN}$ is cut off at the blue end of the $J$ band). The outer boundary of the coma $\left(\sim 10^{5} \mathrm{~km}\right)$ is consistent with the lifetimes of $\mathrm{C}_{2}$ and $\mathrm{CN}$ radicals, and the radiation pressure field at the heliocentric distance of 1.25 AU. The isophote shapes are not in a good accord with the classical "fountain" model (Arpigny 1965), which assumes a small, symmetric nuclear source, but the complex qualitative behavior of icy grains, extending mainly into the fan, which could generate the extended halo, may remove this modest inconsistency. Our ellipse-fitting photometry also shows that the isophote centers move in the NE direction, as one goes toward fainter levels; this is also apparent from Fig. 3.

Note also the prominent asymmetry of the outer isophotes (a "bump"), NW from the nucleus, which is not caused by the plate nonuniformity (Fig. 3). The linear size of this feature is comparable to the turbulence scales in the interplanetary medium (IPM) (Lee and Wu 1979), and may be caused by it. Perhaps a more likely explanation is that the interaction of the radial flow colliding at an oblique angle with the solar radiation field produces some instability. Apparently, our photometry reaches close to the physical limits of the coma, where it dissipates in the IPM. Detailed modeling of such phenomena is beyond the scope of the present report.

\section{A SEARCH FOR EXTRANUCLEAR FEATURES}

The fan/jet feature is very prominent, emerging from the nucleus on the "front" (sunward) side (Fig. 8). The origin of the flow is unresolved on our plates $(\sim 200 \mathrm{~km}$ at the comet). This may indicate that the rotational axis of the comet's nucleus is close to the direction of motion, since any appreciable rotation would likely produce rounder isophotes (the observed isophotes are asymmetric and elongated in the direction of the jet). An alternative is that the ejection takes place in a rather narrow zone, as seen in cometocentric coordinates. It had been suggested earlier (Whipple and Sekanina 1979) that the asymmetric orbital visual-brightness curve of P/Encke may be interpreted as a signature for the latter possibility. However, A'Hearn et al. (1984) show that the light curve in $\mathrm{OH}$ is symmetric, although the light curves in 

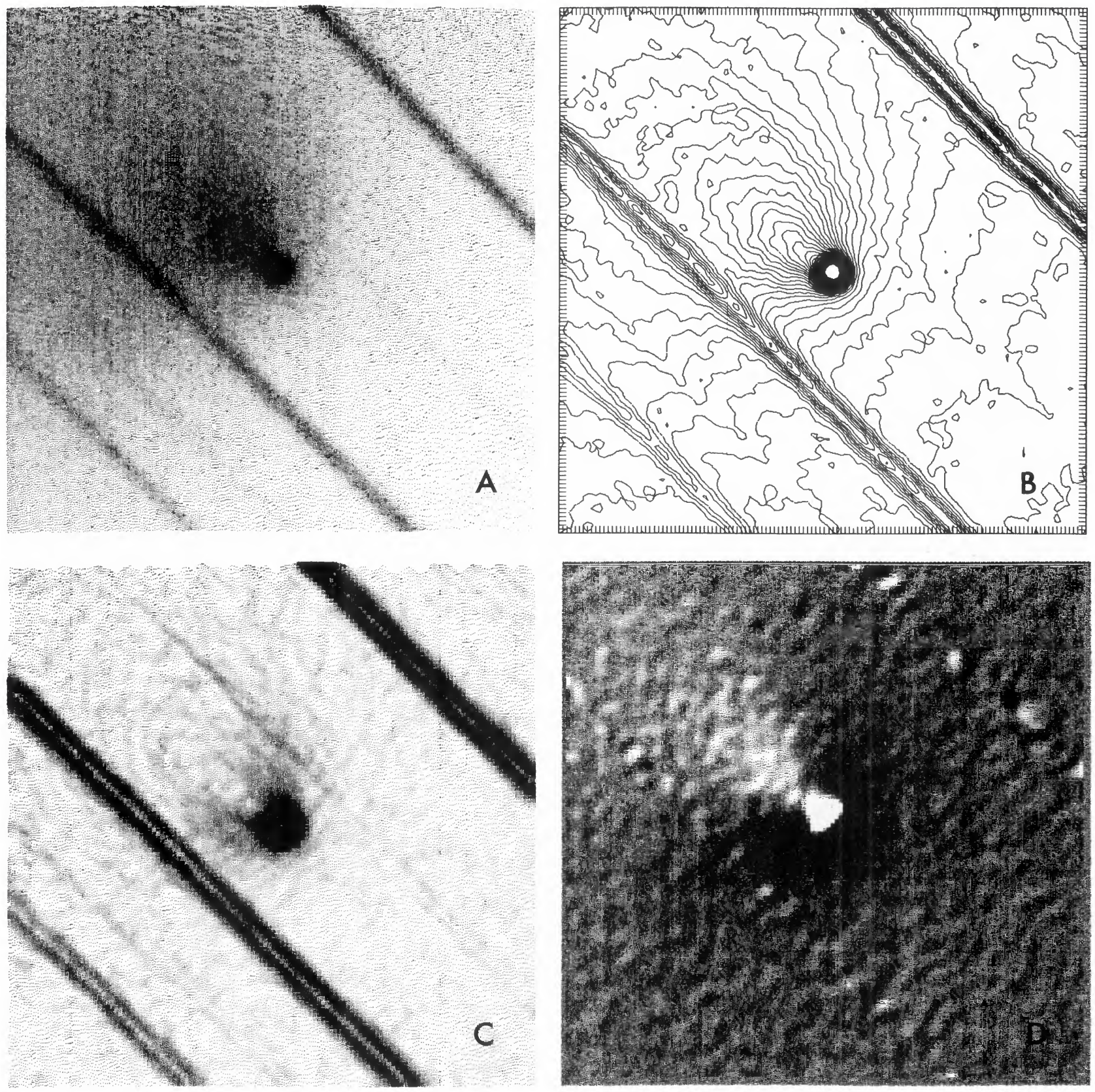

FIG. 8. (A) Central portion of the $J$ image: the fan/jet is very prominent. The field size is $47.6 \mathrm{arcsec}^{2}$, and the orientation is as in Fig. 1. (B) The same, but in a contour map representation: note the very collimated shape of the jet near the nucleus. (C) The same image enhanced with a two-dimensional gradient technique (Laplacean filter); density gradients at the fan edges show up. (D) The image enhanced with a directional gradient technique, along the stellar trails $\left(45^{\circ}\right)$; the fan and the strong density gradient stand out.

$\mathrm{C}_{2}, \mathrm{CN}, \mathrm{CS}$, and the thermal infrared are also asymmetric (Campins and A'Hearn 1984). Clearly, chemical inhomogeneities play an important role, but the existence of the sunward jet is likely responsible for the observed asymmetries in at least some of the light curves.

There is no evidence for bipolarity of the flow. The flow seems to be very collimated, indicating that the outgassing flow velocities are at least a few times larger than the thermal gas velocities, because the broadening of the fan is probably determined by the gas diffusion perpendicular to the flow axis. Very similar, asymmetric morphology was found also in the comet Pons-Winnecke (Sekanina 1981). This is to be contrasted with the dust-ejection phenomena discovered on the old P/Halley plates by Larson and Sekanina (1984), where spiral patterns imply slow rotation and ejection roughly perpendicular to the rotation axis.

The enhanced images (Fig. 8) show very clearly the strong increase in the density gradient at the tail side. We do not see any other imbedded features or detachments in our enhanced images (central parts of which are shown in Fig. 8)the flow seems to be relatively quiescent.

In conclusion, it appears that detailed surface photometry and imaging of comets, in particular when coupled with simultaneous spectroscopy, can yield some valuable information on the structure of these fascinating objects, and the physical processes in them. We close with a plea for future high-angular-resolution imaging and spectroscopy, both in the optical and thermal infrared. 
We would like to thank to Dr. J. Stauffer for help with the Kitt Peak observations. We acknowledge valuable discussions with Drs. Z. Sekanina, M. Hanner, and D. Schleicher. Dr. Z. Sekanina provided most valuable comments and criti- cal reading of the manuscript. $H$. $S$. has been supported by the grants from NSF, NASA, and the California Space Institute. S. D. has been supported in part by the University of California Moore and Regents Fellowships.

\section{REFERENCES}

A'Hearn, M. F. (1982). In Comets, edited by L. L. Wilkening (University of Arizona, Tucson), p. 433.

A'Hearn, M. F., Feldman, P. D., Millis, R. L., and Stewart, A. I. F. (1984). Bull. Am. Astron. Soc. 16, 638.

Arpigny, C. (1965). Mem. R. Acad. Belg. 35, No. 5.

Campins, H., and A'Hearn, M. F. (1984). Bull. Am. Astron. Soc. 16, 638.

Delsemme, A., and Miller, D. C. (1971). Planet. Space Sci. 19, 1229.

Hanner, M. (1981). Icarus 47, 342.

Jewitt, D. C., Soifer, B. T., Neugebauer, G., Mathews, K., and Danielson, G. E. (1982). Astron. J. 87, 1854.
Kent, S. M. (1983). Astrophys. J. 266, 562.

Larson, S., and Sekanina, Z. (1984). Astron. J. 89, 571.

Lee, L. C., and Wu, C. S. (1979). Astrophys. J. 228, 935.

Newburn, R., and Spinrad, H. (1984). Astron. J. 89, 289.

Newburn, R. and Spinrad, H. (1985). Astron. J. (to be submitted).

Schweizer, F., Gonzales, R., and Saa, O. (1980). CTIO News. No. 3, p. 1

Sekanina, Z. (1979). Icarus 37, 420.

Sekanina, Z. (1981). Annu. Rev. Earth Planet. Sci. 9, 113.

Sekanina, Z., and Larson, S. (1984). Astron. J. 89, 1408.

Whipple, F. L., and Sekanina, Z. (1979). Astron. J. 84, 1894. 